\title{
Macht Ungerechtigkeit krank? Gesundheitliche Folgen von Einkommens(un)gerechtigkeit
}

Die Veränderungen auf dem Arbeitsmarkt (Flexibilisierung, Ausweitung des Niedriglohnsektors) und die zunehmend ungleiche Verteilung der Erwerbseinkommen werfen vermehrt Fragen nach der Gerechtigkeit der Löhne in Deutschland auf. Ein beträchtlicher Teil der hiesigen Arbeitnehmer - etwa ein Drittel der abhängig Beschäftigten - empfindet das eigene Erwerbseinkommen als zu niedrig und daher als ungerecht. Dies hat handfeste Konsequenzen für die Betroffenen: Ein als ungerecht empfundenes Erwerbseinkommen kann zu stressbedingten gesundheitlichen Beeinträchtigungen führen. Da das Gefühl, für die eigenen Leistungen nicht adäquat entlohnt zu werden, vor allem bei niedrigqualifizierten Beschäftigten und bei Beschäftigten mit geringen Brutto-Stundenlöhnen vorherrscht, verstärken sich dadurch außerdem gesundheitliche Ungleichheiten. ${ }^{1}$

REINHARD SCHUNCK, CARSTEN SAUER, PETER VALET

\section{Einleitung}

Die Gesundheit wird häufig als „das wichtigste Gut“ bezeichnet, das dem Menschen zur Verfügung steht. Ein schlechter Gesundheitszustand beeinträchtigt etwa die Lebensqualität (Wang et al. 2008) oder die Chancen zur Lebensgestaltung nachhaltig (Chirikos 1993; Hasselhorn/Rauch 2013). Der Gesundheitszustand von Menschen wird neben genetischen Faktoren auch maßgeblich durch deren sozioökonomische Position beeinflusst (Lampert et al. 2007; Richter/Hurrelmann 2007). Hierbei kommt der Position auf dem Arbeitsmarkt eine entscheidende Rolle zu (Siegrist/Theorell 2006). Diese bestimmt sowohl den Zugang zu wichtigen Ressourcen, wie etwa Erwerbseinkommen oder beruflichem Status, als auch die Belastungen und Gefährdungen, denen Beschäftigte ausgesetzt sind. Während körperlich gefährliche Arbeiten und die damit verbundenen Risiken seit Jahren abnehmen, nimmt die Bedeutung von psychosozialen Belastungen in der Arbeitswelt zu (BMAS 2012). Psychosoziale Belastungen entstehen beispielsweise dann, wenn Beschäftigte der Meinung sind, die von ihnen erbrachten Leistungen würden nicht angemessen vergütet (Siegrist/ Theorell 2006), sie also das Gefühl haben, nicht gerecht entlohnt zu werden. Dies trifft auf einen beträchtlichen Anteil von Arbeitnehmern in Deutschland zu: So empfindet etwa ein Drittel der abhängig Beschäftigten die Höhe des eigenen Erwerbseinkommens als ungerecht (Liebig et al. 2010).
Zahlreiche sozialwissenschaftliche Abhandlungen untersuchen die Ursachen dieser Ungerechtigkeitswahrnehmungen. Dabei zeigt sich, dass nicht alle Personengruppen ihr Erwerbseinkommen in gleichem Maße als gerecht oder ungerecht wahrnehmen. Vor allem niedrig qualifizierte Arbeitnehmer, beispielsweise einfache Arbeiter oder Beschäftigte in einfachen Dienstleistungsberufen, empfinden ihr Erwerbseinkommen als ungerechterweise zu gering (Liebig 2007). Mit den Folgen von Ungerechtigkeitswahrnehmungen beschäftigen sich vor allem ökonomische Studien, die die Produktivität von Arbeitnehmern oder deren Fehlverhalten und dadurch verursachte gesamtwirtschaftliche Kosten im Auge haben, sowie psychologische und soziologische Arbeiten, die die Auswirkungen von Ungerechtigkeiten auf die Arbeitszufriedenheit untersuchen (Pritchard et al. 1972; Dittrich et al. 1985; Berkowitz et al. 1987). Einige querschnittlich $^{2}$ angelegte Studien zeigen, dass ein als un-

1 Wir danken Anja Abendroth, Martin Diewald, Katja Freistein, Stefan Liebig, Silvia Melzer, Stephanie Pausch sowie zwei anonymen Gutachtern/Gutachterinnen und der Redaktion der WSI-Mitteilungen für wichtige Hinweise und Anregungen.

2 Querschnittsuntersuchungen sind Studien, bei denen die Teilnehmer zu einem einzigen Zeitpunkt befragt werden. Dagegen werden in Längsschnitt- oder Paneluntersuchungen Befragte mehrmals zu verschiedenen Zeitpunkten in- 
gerecht empfundenes Erwerbseinkommen zu einem Anstieg von Krankheitstagen (Liebig/Schupp 2008a) oder zu einer erhöhten Kündigungsneigung bei den Beschäftigten führen kann (Alexander/Rudermann 2010).

Es fehlen bislang jedoch Untersuchungen dazu, ob als ungerecht empfundene Erwerbseinkommen direkte gesundheitliche Konsequenzen für die betroffenen Beschäftigten haben. Das überrascht umso mehr, da es deutliche theoretische (Siegrist 1996a, 2000; Greenberg 2010) und auch erste empirische (Greenberg 2010; Falk et al. 2011) Hinweise gibt, dass Ungerechtigkeitswahrnehmungen als Stressor durchaus gesundheitsrelevant werden können. Die bisherigen empirischen Untersuchungen lassen jedoch viele Fragen offen, da sie entweder auf Laborexperimenten oder auf Querschnittsuntersuchungen beruhen. So kann einerseits die Generalisierbarkeit der Ergebnisse infrage gestellt werden, insbesondere, wenn es sich um künstliche und kurzfristig „verabreichte“ Ungerechtigkeiten unter Laborbedingungen handelt. Andererseits bleibt die Frage nach der Kausalrichtung unbeantwortet, wenn die Ergebnisse auf Querschnittsanalysen beruhen, da diese Ursache und Wirkung nicht trennen können (Brüderl 2010).

Zur Schließung dieser Lücke will die vorliegende Studie beitragen. Wir haben auf Basis von Daten des Sozio-oekonomischen Panels der Jahre 2005 bis 2011 im Längsschnitt analysiert, wie sich eine als ungerecht empfundene Entlohnung auf die individuelle Gesundheit auswirkt. Die aus dem repräsentativen Datensatz gewonnenen Ergebnisse geben deutliche Hinweise auf eine Verschlechterung des subjektiv wahrgenommenen Gesundheitszustands, wenn das Erwerbseinkommen als zu niedrig empfunden wird. Die Analysen zeigen zudem, dass dies nicht an der tatsächlichen Einkommenshöhe oder der beruflichen Position liegt. Mit anderen Worten: Allein schon, wenn das eigene Erwerbseinkommen als ungerecht empfunden wird, scheint dies einen eigenständigen negativen Effekt auf die Gesundheit zu haben.

\section{Krankheitsentstehung durch Ungerechtigkeitswahrnehmungen}

\subsection{Geben und Nehmen - Erwartungen von Arbeitnehmern}

Im Mittelpunkt der theoretischen Überlegungen steht die Annahme, dass es sich bei einem Beschäftigungsverhältnis zwischen Arbeitnehmer und Arbeitgeber um eine Austauschbeziehung handelt, in welcher der Arbeitnehmer seine Arbeitskraft (Leistung) zur Verfügung stellt und im Gegenzug Vergütungen (beispielsweise Erwerbseinkommen, Beschäftigungssicherheit, Aufstiegsmöglichkeiten, Ansehen, etc.) erhält (Rousseau 1995; Siegrist/Theorell 2006; Chan/
Goldthorpe 2007; Diewald 2007). Der Austauschbeziehung liegt dabei die Erwartung zugrunde, für die erbrachten Leistungen angemessen entlohnt zu werden (Gouldner 1960; Siegrist 1996a, 2000; Siegrist/Theorell 2006). Um die Höhe einer angemessenen, also als gerecht empfundenen Entlohnung zu bestimmen, vergleichen Arbeitnehmer ihre Leistungen und Vergütungen mit denen von Kollegen, Partnern oder anderen konkreten Personen (Adams 1965) oder mit dem, was man üblicherweise mit den eigenen Qualifikationen in einem bestimmten Beruf verdient (Berger et al. 1972). Der Vergleichsstandard kann somit ein „signifikanter" oder ein "generalisierter“ anderer sein. Über diese Vergleiche bilden Beschäftigte sich eine Einschätzung, wie hoch ein gerechtes Erwerbseinkommen sein müsste. Dieses hypothetische, gerechte Erwerbseinkommen bemisst sich also an den erbrachten eigenen Leistungen, an erhaltenen anderen (nicht-monetären) Vergütungen und der Entlohnung von Beschäftigten mit ähnlichen Qualifikationen und Tätigkeiten, wie etwa Arbeitskollegen (Homans 1961; Adams 1965; Walster et al. 1973). Besteht aus Sicht der Beschäftigten ein Gleichgewicht zwischen den erbrachten Leistungen und ihren Qualifikationen auf der einen und den erhaltenen Vergütungen auf der anderen Seite, wird das Erwerbseinkommen als gerecht empfunden. Besteht ein Ungleichgewicht zwischen Leistungen und Vergütungen, so wird das Erwerbseinkommen als ungerecht empfunden (Liebig et al. 2013). Dabei kann man sein Einkommen als ungerechterweise zu hoch oder ungerechterweise zu gering bewerten. Im Folgenden werden wir uns auf die Situation der Unterbezahlung konzentrieren, da nur sehr wenige Befragte angeben, überbezahlt zu sein (Sauer/Valet 2013), und weil im Hinblick auf gesundheitliche Konsequenzen die theoretischen Erklärungen und empirischen Studien auf ungerechte Unterbezahlung abzielen.

\subsection{Die Folgen enttäuschter Erwartungen}

Die Gerechtigkeitsbewertung des eigenen Erwerbseinkommens kann also als eine umfassende Bewertung des Verhältnisses von erbrachten Leistungen und erhaltenen Vergütungen verstanden werden. Denn erstens werden bei der Festlegung des gerechten Erwerbseinkommens andere, nicht-monetäre Vergütungen berücksichtigt und zweitens wählen Beschäftigte, wie bereits erwähnt, keine willkürlichen Beträge, sondern orientieren sich am Erwerbseinkommen anderer (beispielsweise von Kollegen) und beziehen demzufolge den Arbeitskontext mit ein. Wird das eigene Erwerbseinkommen vom Beschäftigten als zu niedrig wahrgenommen, entspricht dies einer sogenannten sozialen Gratifikationskrise (Siegrist 1996b). Der Begriff beschreibt

terviewt. So ist es möglich, Veränderungen (des Gesundheitszustandes) über einen längeren Zeitraum zu beobachten und auf andere Veränderungen (der Gerechtigkeit der Entlohnung) zurückzuführen. 
eine Austauschsituation, in der ein Ungleichgewicht zwischen erbrachten Leistungen und erhaltenen Vergütungen (Gratifikationen) besteht (Siegrist 1996a, 1996b, 2000, 2011). Eine Gratifikationskrise führt zu Stressreaktionen (Siegrist 1996b, 2000; Calnan et al. 2004; Siegrist et al. 2004; Siegrist/ Theorell 2006; Hämmig et al. 2012) - ein Befund, der auch aus der Gerechtigkeitsforschung bekannt ist (Markovsky 1988; Greenberg 2010). Diese Reaktionen erhöhen die Wahrscheinlichkeit, dass stressbedingte seelische und körperliche Erkrankungen auftreten (van Vegchel et al. 2005; Siegrist/ Dragano 2008). So zeigen sich deutliche Zusammenhänge zwischen einem wahrgenommen Ungleichgewicht von erbrachten Leistungen und erhaltenen Vergütungen und dem Auftreten von Krankheiten des Herz-Kreislauf-Systems (Lynch et al. 1997; Bosma et al. 1998; Kivimaki et al. 2002; Kuper et al. 2002; van Vegchel et al. 2005) oder dem Auftreten seelischer Erkrankungen, wie beispielsweise Depressionen oder depressiven Symptomen (Pikhart et al. 2004; Kivimaki et al. 2007; Siegrist 2011).

Im Kontext des Modells sozialer Gratifikationskrisen (auch Effort-Reward-Imbalance-Modell genannt) werden erbrachte Leistungen und erhaltene Vergütungen über ein standardisiertes Messinstrument erhoben, welches sowohl Leistungen als auch Vergütungen auf verschiedenen Dimensionen abbildet (Siegrist et al. 2004). Da ein als ungerecht empfundenes Erwerbseinkommen per Definition einem Ungleichgewicht zwischen Leistungen und erhaltenen Vergütungen entspricht, verwenden wir in dieser Arbeit die Ungerechtigkeitsbewertung des eigenen Einkommens als Indikator für das Vorliegen einer Gratifikationskrise (Falk et al. 2011).

Die zentrale Hypothese lautet, dass ein als zu niedrig empfundenes Erwerbseinkommen eine Gratifikationskrise hervorruft und als Folge den individuellen (seelischen und körperlichen) Gesundheitszustand verschlechtert.

\section{Daten und Methoden}

Die Datengrundlage für die empirischen Analysen bildet das Sozio-oekonomische Panel (SOEP). Das SOEP ist eine repräsentative Wiederholungsbefragung, die seit 1984 jährlich in Deutschland durchgeführt wird (Wagner et al. 2007; SOEP 2012). Die Analysen sind auf die Jahre 2005 bis 2011 beschränkt, da erst seit dem Jahr 2005 die Gerechtigkeitsbewertung des Erwerbseinkommens abgefragt wird. Als abhängige Variable wird die subjektive Gesundheitseinschätzung herangezogen. Die Frage „Wie würden Sie Ihren gegenwärtigen Gesundheitszustand beschreiben?" wird im SOEP jährlich auf einer fünfstufigen Skala erhoben. Dabei wird der Gesundheitszustand zwischen „sehr gut“, „gut“, "zufriedenstellend“, „weniger gut“ und „schlecht“ von den Befragten selbst eingeschätzt. Die Einschätzung des subjektiven Gesundheitszustandes ist ein verlässlicher Indikator für das Krankheits- und Sterberisiko (Idler/Benyamini 1997; Burstrom/Fredlund 2001; DeSalvo et al. 2006) und wird deshalb in der gesundheitssoziologischen und sozialepidemiologischen Forschung häufig verwendet (Kunst et al. 2005; Laaksonen et al. 2006; Eikemo et al. 2008; Espelt et al. 2008). Der Praxis folgend haben wir die ursprünglich fünfstufig skalierte Variable für die Analysen dichotomisiert. Somit haben wir den Wert 1, wenn der Befragte angibt, einen guten oder sehr guten Gesundheitszustand zu haben, und den Wert 0, wenn dies nicht der Fall ist. Dieses Vorgehen haben wir gewählt, da uns einerseits inhaltlich die Differenz von „gesund/nicht gesund“ interessiert und andererseits, weil Studien zeigen, dass der Informationsverlust durch diese Dichotomisierung zu vernachlässigen ist (Manor et al. 2000).

Die zentrale unabhängige Variable ist die subjektive Gerechtigkeitsbewertung des eigenen Erwerbseinkommens. Diese wird im SOEP ab 2005 alle zwei Jahre mit der Frage „Ist das Einkommen, das Sie in Ihrer jetzigen Stelle verdienen, aus Ihrer Sicht gerecht?“ erhoben. Bei Befragten, die ihr Erwerbseinkommen als ungerecht empfinden, wird zudem erfragt, was für sie ein gerechtes Netto- und Brutto-Einkommen wäre. Aus diesen Informationen wurde eine dichotome Variable gebildet, die den Wert 0 annimmt, wenn das tatsächliche Erwerbseinkommen dem als gerecht empfundenen Erwerbseinkommen entspricht, und sie nimmt den Wert 1 an, wenn das tatsächliche Erwerbseinkommen geringer als das als gerecht empfundene Erwerbseinkommen ist. ${ }^{3}$

Weitere Kontrollvariablen für die Vorhersage des Gesundheitszustandes sind Eigenschaften des Beschäftigungsverhältnisses, die von der gesundheitswissenschaftlichen Forschung als relevante Faktoren identifiziert wurden (Lampert et al. 2007; Richter/Hurrelmann 2007), wie das tatsächliche Erwerbseinkommen (Brutto-Stundenlohn), der berufliche Status, ${ }^{4}$ Befristung (Rodriguez 2002), Leih- bzw. Zeitarbeit (Langhoff et al. 2012), die Zufriedenheit mit der Beschäftigung (Faragher et al. 2005) sowie soziodemografische Variablen.

3 In der Gerechtigkeitsforschung wird häufig das kontinuierliche Maß für das Ungerechtigkeitsempfinden nach Jasso (1978, 2007) verwendet (z. B. Liebig/Schupp 2008b; Liebig et al. 2012). Dieses Maß entspricht dem logarithmierten Verhältnis von tatsächlichen und gerechten Erträgen und bildet den mathematischen Kern der Gerechtigkeitstheorie (Jasso/Wegener 1997), ist allerdings in seiner Interpretation weniger eingängig. Deshalb haben wir uns für eine einfache Darstellung (gerecht/ungerecht) entschieden. Die Ergebnisse bleiben unverändert, wenn das metrische Maß verwendet wird.

4 Der berufliche Status wird mittels des International SocioEconomic Index of Occupational Status (ISEI) gemessen (Ganzeboom et al. 1992). Dieser Index kann Werte zwischen 16 (bspw. Reinigungskraft) und 90 (bspw. Richter) annehmen. 
Die Analysen beschränken sich auf erwerbstätige Personen im Alter von 18 bis 65 Jahren. Aus theoretischen Gründen werden Auszubildende und Selbstständige von den Analysen ausgeschlossen: In Ausbildung befindliche Personen erhalten eine festgeschriebene Ausbildungsvergütung, die nicht auf einer Austauschrelation von Arbeit gegen Entgelt beruht; Selbstständige befinden sich nicht in einer Austauschbeziehung mit einem Arbeitgeber. Insgesamt gehen somit 26.533 Beobachtungen in die Analysen ein, die von 12.268 Personen stammen.

Für die Auswertungen der Paneldaten wird ein logistisches random-effects Regressionsmodell geschätzt, bei dem eine sogenannte within- und between-Dekomposition der Effektschätzer vorgenommen wurde (Allison 2009; Schunck 2013; Wooldridge 2010). Dieses auch als Hybrid-Modell (Allison 2009) bekannte Verfahren verbindet die Vorteile von fixed-effects Regressionsmodellen mit denen von random-effects Regressionsmodellen (Schunck 2013). Der Vorteil von fixed-effects Regressionsmodellen besteht darin, dass auf alle zeitkonstanten Drittvariablen (sogenannte zeitkonstante unbeobachtete Heterogenität) kontrolliert wird (Allison 2009; Giesselmann/Windzio 2012; Andreß et al. 2013), unabhängig davon, ob sie einfach beobachtbar sind, wie beispielsweise die abgeschlossene Schullaufbahn oder das Geschlecht, oder schwer zu messen sind, wie fixe biologische Krankheitsdispositionen oder Persönlichkeitseigenschaften. Fixed-effects Modelle können aber den Effekt zeitkonstanter Merkmale, wie bspw. des Geschlechts, nicht schätzen, weil Effekte nur durch intra-individuelle Varianz geschätzt werden. Random-effects Modelle können wiederum die Effekte

ABB.

\section{Erwerbseinkommen und Ungerechtigkeitsempfinden*}

Angaben in Prozent

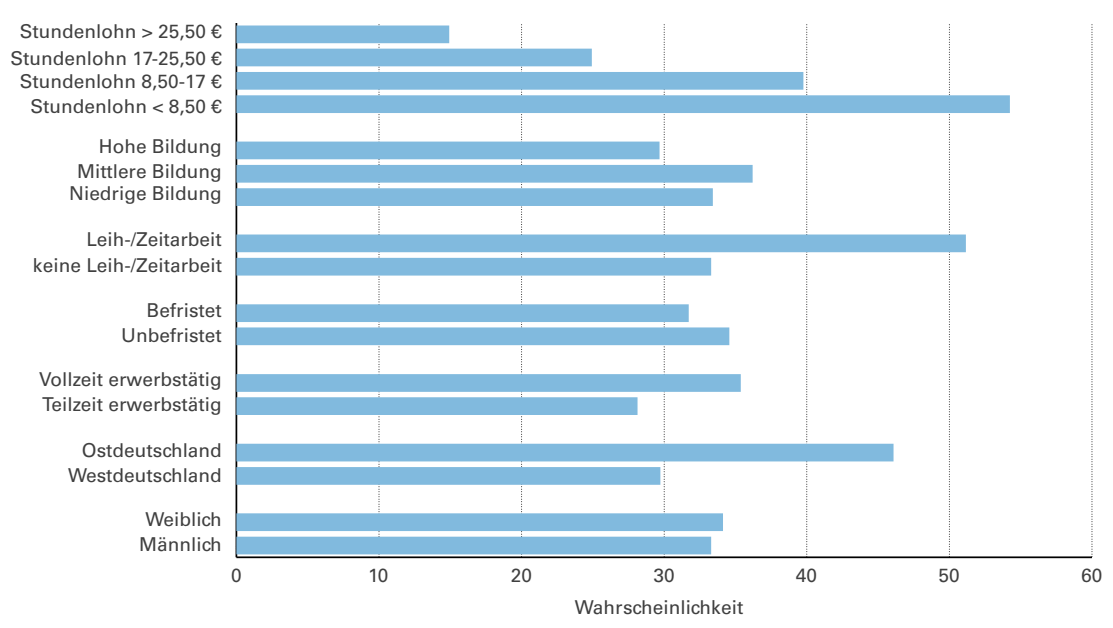

*Die Abbildung zeigt jeweils die geschätzte Wahrscheinlichkeit, das eigene Erwerbseinkommen als ungerechterweise zu niedrig zu empfinden, wenn man der jeweiligen Gruppe angehört. Bis auf Geschlecht sind alle Gruppenunterschiede statistisch signifikant $(\mathrm{p}<.01)$.

Quelle: SOEP v28, 2005-2011, N = 26.533; Berechnungen und Darstellung der Autoren zeitkonstanter Merkmale schätzen, ihre Schätzer sind allerdings nicht bereinigt um zeitkonstante unbeobachtete Heterogenität. Das Hybrid-Verfahren erlaubt es, innerhalb eines Modells für sich verändernde (zeitvariante) Merkmale within-Schätzer (dies entspricht den fixed-effects Schätzern) und zugleich den Effekt unveränderlicher (zeitinvarianter) Merkmale als random-effects Schätzer zu berechnen (Neuhaus/Kalbfleisch 1998; Allison 2009, S. 39ff.; Wooldridge 2010, S. 619f.; Schunck 2013). Im vorliegenden Fall werden Geschlecht und Bildung, bei denen es keine bzw. zu wenig Veränderung über die Zeit gibt, als random-effects Schätzer berechnet. Für alle übrigen Variablen werden within-Schätzer berechnet.

\section{Ergebnisse}

In der folgenden Darstellung der Ergebnisse wird zunächst beschrieben, welche Personen ihr Erwerbseinkommen häufiger oder seltener als ungerecht bewerten. Anschließend wird der Zusammenhang zwischen der Gerechtigkeitswahrnehmung des Erwerbseinkommens und dem Gesundheitszustand genauer betrachtet.

\subsection{Wer ist von Lohnungerechtigkeit betroffen?}

Es zeigt sich, dass die Mehrheit der Befragten (66\%) ihr Erwerbseinkommen als gerecht empfindet. Allerdings liegt der Anteil derer, die ihr Erwerbseinkommen als ungerecht empfinden, bei mehr als einem Drittel (34\%). Diese Befragten sind der Meinung, sie müssten mehr verdienen, damit erbrachte Leistungen und erhaltene Gratifikationen im Gleichgewicht sind. Die Mediandifferenz zwischen als gerecht empfundenem und tatsächlichem Erwerbseinkommen bei Personen, die ihr monatliches Erwerbseinkommen als zu niedrig empfinden, beträgt ca. 27 Prozentpunkte oder $500 €$.

Allerdings unterscheiden sich verschiedene Beschäftigtengruppen hinsichtlich der Bewertung des eigenen Erwerbseinkommens als gerecht bzw. ungerecht. Abbildung 1 verdeutlicht diese Unterschiede. Zunächst zeigen sich auch an dieser Stelle die bekannten Zusammenhänge zwischen der tatsächlichen Höhe des Erwerbseinkommens und der Gerechtigkeitsbewertung des Einkommens (Liebig/Schupp 2008a). Über die Hälfte der Beschäftigten (54,6 \%), deren Brutto-Stundenlohn unter 8,50€ liegt, empfindet ihr Erwerbseinkommen als ungerechterweise zu niedrig. Der Anteil ist deutlich kleiner bei einem Brutto-Stundenlohn von $8,50-17 €(39,7 \%)$ oder von $17-25,50 €(24,95 \%)$. Bei denjenigen, die über 25,50€ pro Stunde verdienen, empfinden nur knapp 14,7 \% ihr Erwerbseinkommen als ungerechterweise zu niedrig. Im Hinblick auf das Bildungsniveau der Beschäftigten zeigen sich ebenso die bekannten Ergebnisse (Liebig 2007; Liebig/Schupp 2008a): Beschäftigte mit einem hohen Bildungsabschluss (Hochschulabschluss) empfinden 
ihr Erwerbseinkommen seltener als zu niedrig als solche mit niedrigeren (höchstens Hauptschulabschluss) oder mit mittleren Bildungsabschlüssen (Mittlere Reife oder (Fach)Abitur). Ein großer Unterschied besteht zwischen Personen, die als Leih- bzw. Zeitarbeiter beschäftigt sind und solchen, die es nicht sind. Über die Hälfte der Beschäftigten mit Leihoder Zeitarbeitsverträgen empfindet ihr Erwerbseinkommen als zu niedrig. Angesichts der Tatsache, dass sie im Vergleich zu regulär beschäftigten Kollegen und Kolleginnen bei gleicher Leistung in der Regel substanziell weniger verdienen, ist dieser Befund leicht zu erklären. Deutlich ist überdies der Unterschied zwischen Teil- und Vollzeitbeschäftigten. Hier zeigt sich, dass Vollzeitbeschäftigte im Vergleich zu Teilzeitbeschäftigten ihr Erwerbseinkommen häufiger als zu niedrig empfinden. Eine Erklärung dafür könnte sein, dass Vollzeitbeschäftigte meist die Hauptverdiener im Haushalt sind und sich deshalb mehr Gedanken über die Gerechtigkeit ihres Erwerbseinkommens machen als Teilzeitbeschäftigte. Zwischen befristet und unbefristet Beschäftigten besteht an sich ein nur geringer Unterschied, jedoch sind es (überraschenderweise) die unbefristet Beschäftigten, die ihr Einkommen häufiger als zu niedrig empfinden. Weniger überraschend ist, dass Beschäftigte aus den neuen Bundesländern ihr Erwerbseinkommen in höherem Maße als zu niedrig empfinden als Beschäftigte aus den alten Bundesländern. Dieser Befund kann aus den immer noch bestehenden Lohndifferenzen zwischen Ost- und Westdeutschland (Statistisches Bundesamt 2010) erklärt werden.

Interessanterweise unterscheiden sich Männer und Frauen nicht hinsichtlich der Bewertung ihres Einkommens: Sowohl ein Drittel der männlichen Beschäftigten als auch ein Drittel der weiblichen Beschäftigten geben an, dass ihr Erwerbseinkommen, gemessen an den Leistungen, die sie erbringen, zu gering sei. Angesichts des sogenannten gender-wage-gaps, also des bekannten Lohnunterschieds zwischen Männern und Frauen (Finke 2011), war dieser Befund nicht erwartbar. Im Kontext der internationalen Forschung passt dieses Ergebnis allerdings ins Muster. Denn diese zeigt, sowohl was die Zufriedenheit als auch die Gerechtigkeitswahrnehmung des eigenen Erwerbseinkommens angeht, trotz der Lohnlücke keine Unterschiede zwischen Frauen und Männern (Crosby 1982; Liebig et al. 2012). Dies wird unter anderem darauf zurückgeführt, dass sich Frauen eher mit anderen Frauen und Männer eher mit anderen Männern vergleichen und deswegen die Lohnlücke zwischen Frauen und Männern nicht in die Bewertung einfließt (Major 1989, 1994). Andere Forscher machen dagegen „doppelte Standards" in der Bemessung von Leistung oder Kompetenz dafür verantwortlich (Foschi 1996, 2000). Dies meint, dass die gleiche Arbeit „,mit zweierlei Maß gemessen wird“; oder sie verweisen auf Unterschiede in der Selbsteinschätzung von Frauen und Männern, was dann auch Unterschiede in den mit der Arbeit verbundenen Ansprüchen erklären würde (Ridgeway/Smith-Lovin 1999; Ridgeway 2001).

Der Blick auf die Gerechtigkeitswahrnehmung unterschiedlicher Beschäftigtengruppen zeigt also, dass es durch- aus erhebliche Unterschiede in der Bewertung des eigenen Erwerbseinkommens gibt und dass vor allem statusniedrige Berufsgruppen und Personen von einem als zu gering empfundenen Erwerbseinkommen betroffen sind.

\subsection{Macht Ungerechtigkeit krank?}

Bei der Analyse des Zusammenhangs zwischen Ungerechtigkeit und Gesundheit ist zunächst festzuhalten, dass ca. $57,4 \%$ der Befragten von einem guten oder sehr guten Gesundheitszustand berichten und ca. 42,7 \% der Befragten ihren Gesundheitszustand als zufriedenstellend, weniger gut oder schlecht einschätzen.

Um der Frage nachzugehen, ob ein Zusammenhang zwischen der Gerechtigkeitsbewertung des eigenen Erwerbseinkommens und der Gesundheit besteht, ist in Abbildung 2 dieser Zusammenhang abgebildet. Auf der Y-Achse ist die Wahrscheinlichkeit abgetragen, dass von einem guten bzw. sehr guten Gesundheitszustand berichtet wird. Auf der X-Achse wird zwischen Beschäftigten unterschieden, die ihr Erwerbseinkommen als gerecht empfinden und solchen, die ihr Erwerbseinkommen als ungerecht bewerten. Der Zusammenhang zwischen dem subjektiven Gesundheitszustand und der Gerechtigkeitsbewertung des Erwerbseinkommens ist dabei „unter Kontrolle" relevanter Eigenschaften des Beschäftigungsverhältnisses und soziodemografischer Merkmale auf Basis der multivariaten Modellschätzung (die Ergebnisse der Modellschätzung sind in Tabelle 1 aufgeführt) dargestellt. ${ }^{5}$ Die Ergebnisse der multivariaten Analyse entspre-

ABB. 2

\section{Gerechtigkeitsbewertung des Erwerbsein- kommens und subjektiver Gesundheitszustand*} Angaben in Prozent

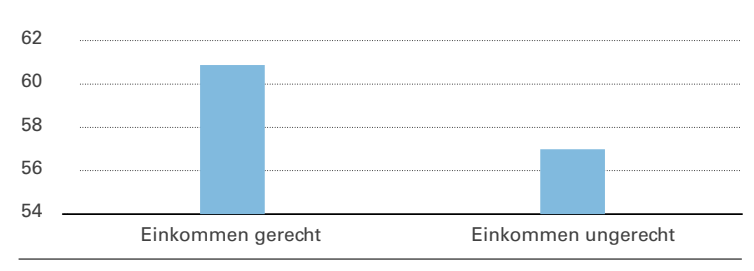

*Die Abbildung zeigt die vorhergesagte Wahrscheinlichkeit, von einem (sehr) guten Gesundheitszustand zu berichten - basierend auf dem in Tabelle 1 präsentierten logistischen Hybrid Regressionsmodell. Unterschied in der vorhergesagten Wahrscheinlichkeit statistisch signifikant $(p<.01)$.

Quelle: SOEP v28, 2005-2011, N = 26.533; Berechnungen und Darstellung der Autoren.

5 Die vorhergesagten Wahrscheinlichkeiten für als gerecht bzw. ungerecht bewertete Erwerbseinkommen ergeben sich dann als gruppenspezifische Mittelwerte der individuellen Wahrscheinlichkeiten (der sogenannte average partial effect) (Wooldridge 2010, S. 22, 577). 
TABELLE 1

Auswirkungen der Gerechtigkeitsbewertung des Einkommens auf den Gesundheitszustand ${ }^{1}$

\begin{tabular}{|c|c|}
\hline Variablen & Odds ratios (OR) \\
\hline Einkommen ungerecht $[1=\mathrm{ja}]$ & $0,805^{* *}$ \\
\hline Arbeitszufriedenheit & $1,237^{* * *}$ \\
\hline Beruflicher Status [ISEI] & 0,996 \\
\hline Brutto Stundenlohn [logarithmiert] & 0,909 \\
\hline Vollzeit erwerbstätig [1 = ja] & 1,012 \\
\hline Arbeitsstunden pro Woche [logarithmiert] & 1,245 \\
\hline Überstunden [1 = ja] & 1,028 \\
\hline Leih-/Zeitarbeit [1 = ja] & $0,621^{*}$ \\
\hline Befristet beschäftigt [1 = ja] & 0,907 \\
\hline Alter & $0,999^{* * *}$ \\
\hline Verheiratet $[1=$ ja] & 1,063 \\
\hline Kinder unter 16 im Haushalt [ $1=$ ja] & 0,892 \\
\hline Geschlecht [1 = männlich] & $1,358^{* * *}$ \\
\hline Bildung niedrig & Referenz \\
\hline Bildung mittel & $1,225^{* *}$ \\
\hline Bildung hoch & $1,695^{* * *}$ \\
\hline Konstante & $0,087^{* * *}$ \\
\hline Personen & 12.268 \\
\hline Beobachtungen & 26.533 \\
\hline Nagelkerke Pseudo $\mathrm{R}^{2}$ & 0,133 \\
\hline
\end{tabular}

${ }^{1}$ Logistisches Hybrid-Regressionsmodell des Gesundheitszustands auf die Gerechtigkeitswahrnehmung und Kontrollvariablen.

Anmerkung: Geschlecht und Bildung als random-effects Schätzer. Modell kontrolliert für Arbeitsplatzwechsel, Perioden (Jahreseffekte) und between-Effekte der zeitveränderlichen Variablen; Odds ratios (OR), Standardfehler robust geschätzt (Bootstrapping, 300 Wiederholungen).

${ }^{*} \mathrm{p}<.05,{ }^{* *} \mathrm{p}<.01,{ }^{* * *} \mathrm{p}<.001$

Quelle: SOEP v28, 2005-2011; Berechnungen der Autoren. jektive Gesundheit (Tabelle 1) - beide Zusammenhänge sind aus der Literatur bekannt (Faragher et al. 2005; Virtanen et al. 2005; Langhoff et al. 2012). Arbeitszufriedenheit wirkt sich positiv auf die subjektive Gesundheit aus (Odds ratio größer 1), während Leih- und Zeitarbeitsverhältnisse gesundheitliche Beeinträchtigungen mit sich bringen (Odds ratio kleiner 1). Interessant ist der Befund, dass weder eine Veränderung der absoluten Einkommenshöhe noch des beruflichen Status (ISEI) einen Effekt auf den Gesundheitszustand haben. Gleichwohl sind Unterschiede in der Gesundheit zwischen Personengruppen mit hohem Erwerbseinkommen und niedrigem Erwerbseinkommen (van Doorslaer/Koolman 2004) und hohem und niedrigem beruflichen Status gut dokumentiert (Dahl 1994; Fujishiro et al. 2010). Dass es in unserer Analyse an statistisch signifikanten Effekten fehlt, lässt sich dadurch erklären, dass sowohl die Einkommenshöhe als auch der berufliche Status und die damit einhergehenden Unterschiede in den Lebenschancen eher zwischen Personengruppen (und gesellschaftlichen Schichten) relevant sind, eine (marginale) Veränderung des Erwerbseinkommens oder des beruflichen Status einer Person zwischen verschiedenen Befragungszeitpunkten aber keinen Effekt besitzt. Bei den Variablen Geschlecht und Bildung zeigt sich, dass einerseits Männer im Vergleich zu Frauen und andererseits Befragte mit höheren und mittleren Bildungsabschlüssen im Vergleich zu Befragten mit niedrigerer Bildung jeweils mit höherer Wahrscheinlichkeit von einem (sehr) guten Gesundheitszustand berichten. Zudem verschlechtert sich der Gesundheitszustand mit voranschreitendem Alter.

Um zu prüfen, ob es geschlechtsspezifische Zusammenhänge zwischen einem als ungerecht empfundenen Erwerbseinkommen und der Gesundheit gibt, wurde das Modell zusätzlich um einen Interaktionseffekt erweitert und es wurden getrennte Modelle für Männer und Frauen berechnet. Es zeigt sich allerdings, dass es keine geschlechtsspezifischen Zusammenhänge gibt: Bei Männern und Frauen scheint sich ein als ungerechterweise zu niedrig empfundenes Erwerbseinkommen gleichermaßen in einem schlechteren Gesundheitszustand niederzuschlagen. Wahrscheinlichkeit, den eigenen Gesundheitszustand als (sehr) gut einzuschätzen, um 3,9 Prozentpunkte auf 57,0 \%. Die geschätzte Veränderung von 3,9 Prozentpunkten entspricht in etwa einer altersbedingten Verschlechterung des Gesundheitszustandes, also einer Verringerung der Wahrscheinlichkeit, einen guten oder sehr guten Gesundheitszustand zu haben, von ca. zwei Jahren im Alter von 40 Jahren, d. h. von 40 auf 42 Jahre. $^{6}$ Dies ist eine durchaus bemerkenswerte Veränderung des subjektiven Gesundheitszustandes, insbesondere wenn man berücksichtigt, dass dies der Effekt einer Einstellung bzw. Bewertung ist und die tatsächliche Einkommenshöhe und weitere Drittvariablen kontrolliert sind.

Neben der Gerechtigkeitswahrnehmung zeigen die Variablen „Zufriedenheit mit dem Beruf“ und „Leih- bzw. Zeitarbeit" statistisch signifikante Effekte auf die sub-

\section{Zusammenfassung und Fazit}

Der vorliegende Beitrag liefert deutliche Hinweise darauf, dass es einen systematischen Zusammenhang zwischen der Gerechtigkeitsbewertung des eigenen Erwerbseinkommens

6 Die altersbedingte Veränderung des Gesundheitszustandes wurde mit der gesamten Stichprobe des SOEP durch ein logistisches random-effects Regressionsmodell geschätzt. 
und der subjektiven Gesundheit gibt. Anknüpfend an das Konzept der sozialen Gratifikationskrise (Siegrist 1996b; Falk et al. 2011) wurde argumentiert, dass ein als zu niedrig empfundenes Erwerbseinkommen auf ein Ungleichgewicht zwischen Aufwand und Ertrag im Arbeitsverhältnis hindeutet, was Stress erzeugt und sich dadurch negativ auf die Gesundheit auswirken kann (Markovsky 1988; Calnan et al. 2004; Siegrist/Dragano 2008; Hämmig et al. 2012). Der geschätzte Einfluss ist dabei durchaus bemerkenswert - er entspricht in seiner Größe ungefähr einer altersbedingten Verschlechterung der Gesundheit von 40 auf 42 Jahre.

Bisher gab es hierzu keine Studien, die sowohl repräsentative Daten als auch adäquate Methoden der Längsschnittdatenanalyse verwenden. Der vorliegende Beitrag zeigt, dass erfahrene Ungerechtigkeiten auch außerhalb künstlicher Laborsituationen gesundheitsrelevante Konsequenzen nach sich ziehen können. Es soll aber nicht versäumt werden, auf einige kritische Punkte der vorliegenden Untersuchung hinzuweisen. Das zugrunde liegende theoretische Modell ist ein stress-theoretisches Modell, gleichwohl steht bei den vorliegenden Daten keine Stressmessung zur Verfügung. Zukünftige Untersuchungen würden davon profitieren, diese theoretische Annahme bei der empirischen Prüfung direkt zu berücksichtigen. Darüber hinaus sollten die Befunde mit anderen Messungen von Gesundheit bzw. Krankheit einer erneuten Überprüfung unterzogen werden. Obwohl der subjektive Gesundheitszustand ein verlässliches und viel genutztes Maß ist (Idler/Benyamini 1997; Burstrom/Fredlund 2001; Kunst et al. 2005; DeSalvo et al. 2006; Laaksonen et al. 2006; Richter/Hurrelmann 2007; Eikemo et al. 2008; Espelt et al. 2008), liegt es nahe, mit objektiveren Methoden (z. B. über klinischen Skalen oder Biomarker) zu erfassen, welche Erkrankungen oder gesundheitlichen Einschränkungen infolge von Ungerechtigkeitswahrnehmungen auftreten.

Bezogen auf die Frage, inwieweit der Befund dieser Untersuchung im Hinblick auf die beträchtliche gesundheitliche Ungleichheit in Deutschland (Lampert et al. 2007; Richter/Hurrelmann 2007) relevant ist, unterstreichen die gezeigten Ergebnisse, dass bei der Entstehung gesundheitlicher Ungleichheit nicht nur materielle Faktoren eine Rolle spielen, sondern dass auch (Ungerechtigkeits)Erfahrungen zur ungleichen Verteilung von Gesundheit und Lebenserwartung beitragen. Da über ein Drittel der abhängig Beschäftigten ihr Erwerbseinkommen als ungerechterweise zu niedrig erachten (siehe auch Liebig et al. 2010), wird deutlich, dass dies keineswegs ein randständiges Phänomen ist. Bei all diesen Personen steht zu befürchten, dass die Ungerechtigkeitserfahrung auch gesundheitliche Auswirkungen hat.

Selbstredend beruht gesundheitliche Ungleichheit auf verschiedenen Ursachen (Richter/Hurrelmann 2007). So korrespondiert gesundheitliche Ungleichheit mit anderen Formen sozialer Ungleichheit wie etwa Bildungsungleichheit, Einkommensungleichheit und Ungleichheit in der beruflichen Stellung. Bestimmte Personengruppen können daher mehrfach benachteiligt sein, wenn bei ihnen (gesundheitliche) Risikofaktoren gehäuft auftreten - dies trifft vor allem bei Personen mit niedriger sozio-ökonomischer Position zu. Dies zeigt sich deutlich bei Personen mit niedrigem Erwerbseinkommen. Über die Hälfte aller Befragten, deren Brutto-Stundenlohn unter 8,50 € liegt, empfindet das eigene Erwerbseinkommen als ungerechterweise zu niedrig. In den höheren Einkommensgruppen sind die Anteile derer, die ihr Erwerbseinkommen als zu niedrig empfinden, deutlich kleiner. Ein niedriger Stundenlohn kann - angesichts des Zusammenhangs von finanziellen Ressourcen und Gesundheit (Lampert et al. 2007) - für sich genommen bereits gesundheitliche Folgen nach sich ziehen. Darüber hinaus sind Beschäftigte mit niedrigen Erwerbseinkommen häufiger von den gesundheitlichen Folgen der Ungerechtigkeitserfahrung betroffen. Ebenso zeigt sich dies bei Beschäftigten mit Leihoder Zeitarbeitsverträgen. Diese Beschäftigtengruppe verdient bei gleicher Tätigkeit oftmals substanziell weniger als ihre im Normalarbeitsverhältnis beschäftigten Kolleginnen und Kollegen. Zusätzlich führen Einkommensvergleiche mit der Stammbelegschaft dazu, dass das eigene Erwerbseinkommen als zu niedrig und somit als ungerecht empfunden wird. Leih- oder Zeitarbeitnehmer sind daher zusätzlich von den gesundheitlichen Konsequenzen dieser Ungerechtigkeitserfahrung betroffen. Bei der Leih- und Zeitarbeit ergibt sich darüber hinaus ein hohes psychisches Belastungsniveau (Langhoff et al. 2012), das sich ebenfalls negativ auf die Gesundheit auswirken kann. Auch unsere Analysen zeigen, dass die Beschäftigung als Leih- oder Zeitarbeiter auch unter Kontrolle möglicher intervenierender Faktoren (wie dem Erwerbseinkommen) noch einen eigenständigen negativen Effekt auf die Gesundheit zu haben scheint.

Es schließen sich an dieser Stelle einige Fragen an, insbesondere, ob die voranschreitende Einkommensspreizung, die sich seit einigen Jahren beobachten lässt (Giesecke/Verwiebe 2008), Auswirkungen auf die Gerechtigkeitsbewertung des eigenen Erwerbseinkommens hat. Des Weiteren stellt sich die Frage, ob eine Zunahme von Einkommensungleichheit auch zu einer Zunahme gesundheitlicher Ungleichheit führt. 


\section{LITERATUR}

Adams, J. S. (1965): Inequity and social exchange, in: Berkowitz, L. (Hrsg.): Advances in Experimental Social Psychology, New York, S. 267-299 Alexander, S./Ruderman, M. (1987): The role of procedural and distribute justice in organizational behavior, in: Social Justice Research 1 (2), S. $177-198$

Allison, P. D. (2009): Fixed Effects Regression Models, Los Angeles Andreß, H.-J./Golsch, K./Schmidt, A. (2013): Applied panel data analysis for economic and social surveys, Heidelberg

Berger, J./Zelditch, M./Anderson, B./Cohen, B. P. (1972): Structural Aspects of Distributive Justice. A Status Value Formulation, in: Berger, J./Zelditch, M./Anderson, B. (Hrsg.): SociologicalTheories in Progress, Boston, S. 119-146 Berkowitz, L./Fraser, C./Treasure, F. P./Cochran, S. (1987): Pay, equity, job gratifications, and comparisons in pay satisfaction, in: Journal of Applied Psychology 72 (4), S. 544-551

Bundesministerium für Arbeit und Soziales (BMAS) (2012): Sicherheit und Gesundheit bei der Arbeit 2010 - Unfallverhütungsbericht Arbeit, Berlin Bosma, H./Peter, R./Siegrist, J./Marmot, M. (1998): Two alternative job stress models and the risk of coronary heart disease, in: American Journal of Public Health 88 (1), S. 68-74

Brüderl, J. (2010): Kausalanalyse mit Paneldaten, in: Wolf, C./Best, H. (Hrsg.): Handbuch der sozialwissenschaftlichen Datenanalyse, Wiesbaden, S. 963-994 Burstrom, B./Fredlund, P. (2001): Self rated health. Is it as good a predictor of subsequent mortality among adults in lower as well as in higher social classes?, in: Journal of Epidemiology and Community Health 55 (11), S. $836-840$

\section{Calnan, M./Wadsworth, E./May, M./Smith, A./Wainwright, D. (2004): Job} strain, effort-reward imbalance, and stress at work: competing or complementary models?, in: Scandinavian Journal of Public Health 32 (2), S. 84-93 Chan, T. W./Goldthorpe, J. H. (2007): Class and status. The conceptual distinction and its empirical relevance, in: American Sociological Review 72 (4), S. $512-532$

Chirikos, T. N. (1993): The Relationship between Health and Labor-Market Status, in: Annual Review of Public Health 14 (11), S. 293-312

Crosby, F. J. (1982): Relative deprivation and working women, New York Dahl, E. (1994): Social Inequalities in III-Health. The Significance of Occupational-Status, Education and Income - Results From a Norwegian Survey, in: Sociology of Health \& IIIness 16 (5), S. 644-667

DeSalvo, K. B./Bloser, N./Reynolds, K./He, J./Muntner, P. (2006): Mortality prediction with a single general self-rated health question, in: Journal of General Internal Medicine 21 (3), S. 267-275

Diewald, M. (2007): Arbeitsmarktungleichheiten und die Verfügbarkeit von So zialkapital, in: Franzen, A./Freitag, M. (Hrsg.): Sozialkapital. Theoretische Grundlagen und empirische Befunde, in: Kölner Zeitschrift für Soziologie und Sozialpsychologie, Sonderheft 47, S. 183-210

Dittrich, J. E./Daniel Couger, J./Zawacki, R. A. (1985): Perceptions of equity, job satisfaction, and intention to quit among data processing personnel, in: Information \& Management 9 (2), S. 67-75

Eikemo, T. A./Huisman, M./Bambra, C./Kunst, A. E. (2008): Health inequalities according to educational level in different welfare regimes: a comparison of 23 European countries, in: Sociology of Health \& IIIness 30 (4) S. 565-582

Espelt, A./Borrell, C./Rodriguez-Sanz, M./Muntaner, C./Pasarin, M. I./Benach, J./Schaap, M./Kunst, A. E./Navarro, V. (2008): Inequalities in health by social class dimensions in European countries of different political traditions, in: International Journal of Epidemiology 37 (5), S. 1095-1105

Falk, A./Menrath, I./Verde, P. E./Siegrist, J. (2011): Cardiovascular Conse quences of Unfair Pay: DIW Berlin, SOEPpapers (380), Berlin

Faragher, E. B./Cass, M./Cooper, C. L. (2005): The relationship between job sat isfaction and health: a meta-analysis, in: Occupational and Environmenta Medicine 62 (2), S. 105-112

Finke, C. (2011): Verdienstunterschiede zwischen Männern und Frauen, in: Wirtschaft und Statistik (1) Januar, S. 36-48

Foschi, M. (1996): Double standards in the evaluation of men and women, in: Social Psychology Quarterly 59 (3), S. 237-254

Foschi, M. (2000): Double standards for competence:Theory and research, in: Annual Review of Sociology 26, S. 21-42

Fujishiro, K./Xu, J./Gong, F. (2010): What does "occupation" represent as an indicator of socioeconomic status? Exploring occupational prestige and health, in: Social Science \& Medicine 71 (12), S. 2100-2107
Ganzeboom, H. B. G./Degraaf, P. M./Treiman, D. J./Deleeuw, J. (1992): A Standard International Socioeconomic Index of Occupational-Status, in: Social Science Research 21 (1), S. 1-56

Giesecke, J./Verwiebe, R. (2008): Die Lohnentwicklung in Deutschland zwischen 1998 und 2005 - Wachsende Ungleichheit, in: WSI-Mitteilungen 61 (2) S. 85-91, http://www.boeckler.de/wsimit_2008_02_giesecke.pdf

Giesselmann, M./Windzio, M. (2012): Regressionsmodelle zur Analyse von Paneldaten, Wiesbaden

Gouldner, A. W. (1960): The Norm of Reciprocity - a Preliminary Statement, in: American Sociological Review 25 (2), S. 161-178

Greenberg, J. (2010): Organizational Injustice as an Occupational Health Risk, in: The Academy of Management Annals 4 (1), S. 205-243

Hämmig, O./Brauchli, R./Bauer, G. F. (2012): Effort-reward and work-life imbalance, general stress and burnout among employees of a large public hospital in Switzerland. in: Swiss Medical Weekly (142), w13577, http://dx.doi. org/10.4414/smw.2012.13577 (letzter Zugriff: 23.09.2013)

Hasselhorn, H. M./Rauch, A. (2013): Perspektiven von Arbeit, Alter, Gesundheit und Erwerbsteilhabe in Deutschland, in: Bundesgesundheitsblatt - Gesundheitsforschung - Gesundheitsschutz 56 (3), S. 339-347

Homans, G. C. (1961): Social Behavior. Its elementary forms, New York Idler, E. L./Benyamini, Y. (1997): Self-rated health and mortality. A review of twenty-seven community studies, in: Journal of Health and Social Behavior 38 (1), S. $21-37$

Jasso, G. (1978): On the justice of earnings. A new specification of the justice evaluation function, in: American Journal of Sociology 83 (6), S. 1398-1419 Jasso, G. (2007): Studying Justice. Measurement, estimation, and analysis of the actual reward and the just reward, in:Törnblom, K./Vermunt, R. (Hrsg.): Distributive and procedural justice, London

Jasso, G./Wegener, B. (1997): Methods for empirical justice analysis. Part 1. Framework, models, and quantities, in: Social Justice Research 10 (4), S. $393-430$

Kivimaki, M./Leino-Arjas, P./Luukkonen, R./Riihimaki, H./Vahtera, J./Kirjonen J. (2002): Work stress and risk of cardiovascular mortality: prospective cohort study of industrial employees, in: British Medical Journal 325 (7369),

S. $857-860$

Kivimaki, M./Vahtera, J./Elovainio, M./Virtanen, M./Siegrist, J. (2007): Effortreward imbalance, procedural injustice and relational injustice as psychosocial predictors of health: complementary or redundant models?, in: Occupational and Environmental Medicine 64 (10), S. 659-665

Kunst, A. E./Bos, V./Lahelma, E./Bartley, M./Lissau, I./Regidor, E./Mielck, A./ Cardano, M./Dalstra, J. A. A./Geurts, J. J. M./Helmert, U./Lennartsson, C./ Ramm, J./Spadea, T./Stronegger, W. J./Mackenbach, J. P. (2005): Trends in socioeconomic inequalities in self-assessed health in 10 European countries, in: International Journal of Epidemiology 34 (2), S. 295-305

Kuper, H./Singh-Manoux, A./Siegrist, J./Marmot, M. (2002): When reciprocity fails: effort-reward imbalance in relation to coronary heart disease and health functioning within the Whitehall II study, in: Occupational and Environmental Medicine 59 (11), S. 777-784

Laaksonen, M./Rahkonen, O./Martikainen, P./Lahelma, E. (2006): Associations of psychosocial working conditions with self-rated general health and mental health among municipal employees, in: International Archives of Occupational and Environmental Health 79 (3), S. 205-212

Lampert, T./Kroll, L. E./Dunkelberg, A. (2007): Soziale Ungleichheit der Lebenserwartung in Deutschland, in: Aus Poltik und Zeitgeschichte 57 (42), S. 11-18 Langhoff, T./Krietsch, I./Schubert, A. (2012): Anforderungen an eine gesundheitsgerechte Gestaltung der Leiharbeit, in: WSI-Mitteilungen 65 (6), S. 464-470, http://www.boeckler.de/wsimit 2012 06 langhoff.pdf Liebig, S. (2007): Modelle und Befunde der empirischen Gerechtigkeitsforschung in Deutschland am Beispiel der Einkommens- und Steuergerechtigkeit, in: Empter, S./Vehrkamp, R. B. (Hrsg.): Soziale Gerechtigkeit - eine Bestandsaufnahme, Bielefeld, S. 111-135

Liebig, S./Sauer, C./Schupp, J. (2012): The justice of earnings in dual-earner households, in: Research in Social Stratification and Mobility 30 (2), S. $219-232$

Liebig, S./Sauer, C./Valet, P. (2013): Gerechtigkeit, in: Mau, S./Schöneck-Voß, N. (Hrsg.): Handwörterbuch zur Gesellschaft Deutschlands, Wiesbaden, S. $286-299$

Liebig, S./Schupp, J. (2008a): Immer mehr Erwerbstätige empfinden ihr Einkommen als ungerecht, in: DIW Wochenbericht 75 (31), S. 434-440 Liebig, S./Schupp, J. (2008b): Leistungs- oder Bedarfsgerechtigkeit? Über ei nen normativen Zielkonflikt des Wohlfahrtsstaats und seiner Bedeutung für 
die Bewertung des eigenen Erwerbseinkommens, in: Soziale Welt 59 (1), S. $7-30$

Liebig, S./Valet, P./Schupp, J. (2010): Wahrgenommene Einkommensgerechtigkeit konjunkturabhängig, in: DIW-Wochenbericht 77 (27-28), S. 11-19 Lynch, J./Krause, N./Kaplan, G. A./Tuomilehto, J./Salonen, J. T. (1997): Workplace conditions, socioeconomic status, and the risk of mortality and acute myocardial infarction. The Kuopio Ischemic Heart Disease Risk Factor Study, in: American Journal of Public Health 87 (4), S. 617-622

Major, B. (1989): Predicting career-entry pay expectations: The role of gender-based comparison, in: Journal of Business and Psychology 3 (3), S. $331-340$

Major, B. (1994): From social inequality to personal entitlement. The role of social comparisons, legitimacy appraisals, and group membership, in: Zanna, M. P. (Hrsg.): Advances in Experimental Social Psychology, New York, S. 293-355 Manor, O./Matthews, S./Power, C. (2000): Dichotomous or categorical response? Analysing self-rated health and lifetime social class, in: International Journal of Epidemiology 29 (1), S. 149-157

Markovsky, B. (1988): Injustice and arousal, in: Social Justice Research 2 (3), S. 223-233

Neuhaus, J. M./Kalbfleisch, J. D. (1998): Between- and within-cluster covariate effects in the analysis of clustered data, in: Biometrics 54 (2), S. 638-645 Pikhart, H./Bobak, M./Pajak, A./Malyutina, S./Kubinova, R./Topor, R./Sebakova, H./Nikitin, Y./Marmot, M. (2004): Psychosocial factors at work and depression in three countries of Central and Eastern Europe, in: Social Science \& Medicine 58 (8), S. 1475-1482

Pritchard, R. D./Dunnette, M. D./Gorgenson, D. O. (1972): Effects of perceptions of equity and inequity on worker performance and satisfaction, in: Journal of Applied Psychology 56 (1), S. 75-94

Richter, M./Hurrelmann, K. (2007): Warum die gesellschaftlichen Verhältnisse krank machen, in: Aus Poltik und Zeitgeschichte 57 (42), S. 3-10

Ridgeway, C. L. (2001): Gender, status, and leadership, in: Journal of Social Issues 57 (4), S. $637-655$

Ridgeway, C. L./Smith-Lovin, L. (1999): The gender system and interaction, in: Annual Review of Sociology 25, S. 191-216

Rodriguez, E. (2002): Marginal employment and health in Britain and Germany: does unstable employment predict health?, in: Social Science \& Medicine 55 (6), S. $963-979$

Rousseau, D. M. (1995): Psychological Contracts in Organizations. Understanding Written and Unwritten Agreements, Thousand Oaks, CA

Sauer, C./Valet, P. (2013): Less is Sometimes More. Consequences of Overpayment on Job Satisfaction and Absenteeism, in: Social Justice Research 26 (2), S. $132-150$

Schunck, R. (2013): Within- and Between-Estimates in Random Effects Models. Advantages and Drawbacks of Correlated Random Effects and Hybrid Models, in: Stata Journal 13 (1), S. 65-76

Siegrist, J. (1996a): Adverse health effects of high-effort/low-reward conditions, in: Journal of Occupational Health Psychology 1 (1), S. $27-41$

Siegrist, J. (1996b): Soziale Krisen und Gesundheit, Göttingen

Siegrist, J. (2000): Place, social exchange and health: proposed sociological framework, in: Social Science \& Medicine 51 (9), S. 1283-1293

Siegrist, J. (2011): Berufliche Gratifikationskrisen und depressive Störungen, in: Psychotherapeut 56 (1), S. 21-25

Siegrist, J./Dragano, N. (2008): Psychosocial stress and disease risks in occupational life. Results of international studies on the demand-control and the effort-reward imbalance models, in: Bundesgesundheitsblatt - Gesundheitsforschung - Gesundheitsschutz 51 (3), S. 305-312

Siegrist, J./Starke, S./Chandola, T./Godin, I./Marmot, M./Niedhammer, I./

Peter, R. (2004): The measurement of effort-reward imbalance at work. European comparisons, in: Social Science \& Medicine 58 (8), S. 1483-1499
Siegrist, J./Theorell, T. (2006): Socio-economic position and health. The role of work and employment, in: Siegrist, J./Marmot, M. (Hrsg.): Social Inequalities in Health. New evidence and policy implications, Oxford, S. 73-97

Sozio-oekonomisches Panel (SOEP) (2012): Daten der Jahre 1984-2011, Version 28., doi:10.5684/soep.v28

Statistisches Bundesamt (2010): 20 Jahre Deutsche Einheit. Wunsch oder Wirklichkeit, Wiesbaden

van Doorslaer, E./Koolman, X. (2004): Explaining the differences in incomerelated health inequalities across European countries, in: Health Economics 13 (7), S. $609-628$

van Vegchel, N./de Jonge, J./Bosma, H./Schaufeli, W. (2005): Reviewing the effort-reward imbalance model: drawing up the balance of 45 empirical studies, in: Social Science \& Medicine 60 (5), S. 1117-1131

Virtanen, M./Kivimaki, M./Joensuu, M./Virtanen, P./Elovainio, M./Vahtera, J. (2005): Temporary employment and health: a review, in: International Journal of Epidemiology 34 (3), S. 610-622

Wagner, G. G./Frick, J. R./Schupp, J. (2007): The german socio-economic panel study (SOEP) - Scope, evolution and enhancements, in: Schmollers Jahrbuch 127 (1), S. 139-169

Walster, E./Berscheid, E./Walster, G. W. (1973): New directions in equity research, in: Journal of Personality and Social Psychology 25 (2), S. 151-176 Wang, H. M./Beyer, M./Gensichen, J./Gerlach, F. M. (2008): Health-related quality of life among general practice patients with differing chronic diseases in Germany. Cross sectional survey, in: BMC Public Health 8 (246), http://www. biomedcentral.com/content/pdf/1471-2458-8-246.pdf (letzter Zugriff: 23.09.2013) Wooldridge, J. M. (2010): Econometric analysis of cross section and panel data, Cambridge, Mass.

\section{AUTOREN}

REINHARD SCHUNCK, Dr., ist Akademischer Rat am Arbeitsbereich Sozialstruktur und soziale Ungleichheit an der Universität Bielefeld. Arbeitsschwerpunkte: Sozialstrukturanalyse, Lebenslaufforschung, Gesundheitssoziologie, Methoden der empirischen Sozialforschung.

reinhard.schunck@uni-bielefeld.de

CARSTEN SAUER, M.A., ist Wissenschaftlicher Mitarbeiter im Sonderforschungsbereich 882 „Von Heterogenitäten zu Ungleichheiten“, Teilprojekt A6 "Die Legitimation von Ungleichheiten: Strukturelle Bedingungen von Gerechtigkeitseinstellungen über den Lebensverlauf" an der Universität Bielefeld. Arbeitsschwerpunkte: Empirische Gerechtigkeitsforschung, soziale Ungleichheit, Methoden der empirischen Sozialforschung.

carsten.sauer@uni-bielefeld.de

PETER VALET, Dipl. Soz.-Wiss., ist Wissenschaftlicher Mitarbeiter am Arbeitsbereich Sozialstruktur und soziale Ungleichheit an der Universität Bielefeld. Arbeitsschwerpunkte: Empirische Gerechtigkeitsforschung, Arbeitsmarktforschung, soziale Ungleichheit.

peter.valet@uni-bielefeld.de 